\title{
Elevation of Plasma Neurophysin in Women on Oral Contraceptives
}

\author{
Alan G. Robinson \\ From the Department of Medicine, University of Pittsburgh, \\ Pittsburgh, Pennsylvania 15261
}

A в S T R A C T The effect of oral contraceptives on the neurohypophysis was demonstrated by changes in the plasma level of a posterior pituitary protein, neurophysin. Neurophysins are intraneuronal proteins associated with oxytocin and vasopressin. They have been shown to be released into the bloodstream. The resting plasma level of neurophysin in normal nonpregnant women is $0.69 \mathrm{ng} /$ $\mathrm{ml} \pm 0.7 \mathrm{SD}$. In women on oral contraceptives, the plasma level is $6.4 \mathrm{ng} / \mathrm{ml} \pm 4.2 \mathrm{SD}(P<0.001)$. Estrogen rather than progesterone causes the elevated neurophysin. The effect is observed within 12-24 h of estrogen administration and disappears 3-11 days after estrogen is discontinued.

The results indicate that oral contraceptives act on the neurohypophysis and that estrogen is a potent pharmacologic stimulus useful in studying synthesis and release of neurophysin.

\section{INTRODUCTION}

The common use of oral contraceptives has stimulated numerous investigations of their effects on ovulation and side effects on other body functions (1-3). While it is generally accepted that the major contraceptive action of the oral agents is by negative feedback on the hypothalamus and the anterior pituitary (4), it is also known that these hormones induce numerous metabolic changes, which have been studied in detail (1-5). This paper reports a newly discovered effect of oral contraceptives on the neurohypophysis. This effect is demonstrated by

Received for publication 30 May 1973 and in revised form 1 April 1974. changes in the plasma level of a posterior pituitary protein, neurophysin.

Neurophysins are present in the neurohypophysis in association with the peptide hormones oxytocin and vasopressin and are thought to act as intraneuronal carrier proteins for these hormones (6). The recent development of radioimmunoassays for neurophysins has allowed the demonstration of the release of these proteins into the bloodstream (7-10). While most animals have two main neurophysins in their pituitaries (10), most immunoassays do not distinguish these two in plasma. We have previously described an assay for human neurophysin (9) and demonstrated elevation of plasma neurophysin in women during pregnancy (11). In samples from nonpregnant women, neurophysin was found to be elevated in those on oral contraceptives. This finding has been investigated in detail, and the results are reported here. Since the collection of these data, a report has appeared in the literature that demonstrates that estrogen administration to normal men will increase plasma neurophysin (12).

\section{METHODS}

The radioimmunoassay of human neurophysin in unextracted plasma, as previously described (9), was used to assay the samples from women. This assay does not distinguish between different human neurophysins. Some later samples from men were assayed by a modification of this assay that used a new antiserum to human neurophysin. Bloods were collected at mid-morning from normal volunteers on ad libitum fluid intake. Samples were collected in heparinized tubes, and the plasma was separated and stored frozen for assay. Several different oral contraceptive agents were being used by the 25 women who provided samples for 
these studies. All contraceptives contained some estrogenic compound. As plasma neurophysin was elevated in women on every agent, no distinction was made between the various oral contraceptives.

\section{RESULTS}

Neurophysin in normal women on no medication was determined in 260 samples from 30 women. This included daily samples throughout the menstrual cycle of five women, hourly samples over a 12 -h period in two women, and mid-morning samples in the other women. The mean plasma neurophysin in normal women was $0.69 \mathrm{ng} / \mathrm{ml} \pm 0.7 \mathrm{SD}$. Neurophysin in women taking oral contraceptives was determined from 196 plasma samples from 25 women. Daily samples were obtained for one cycle from five women, and three weekly samples were obtained from 20 additional women. The mean plasma

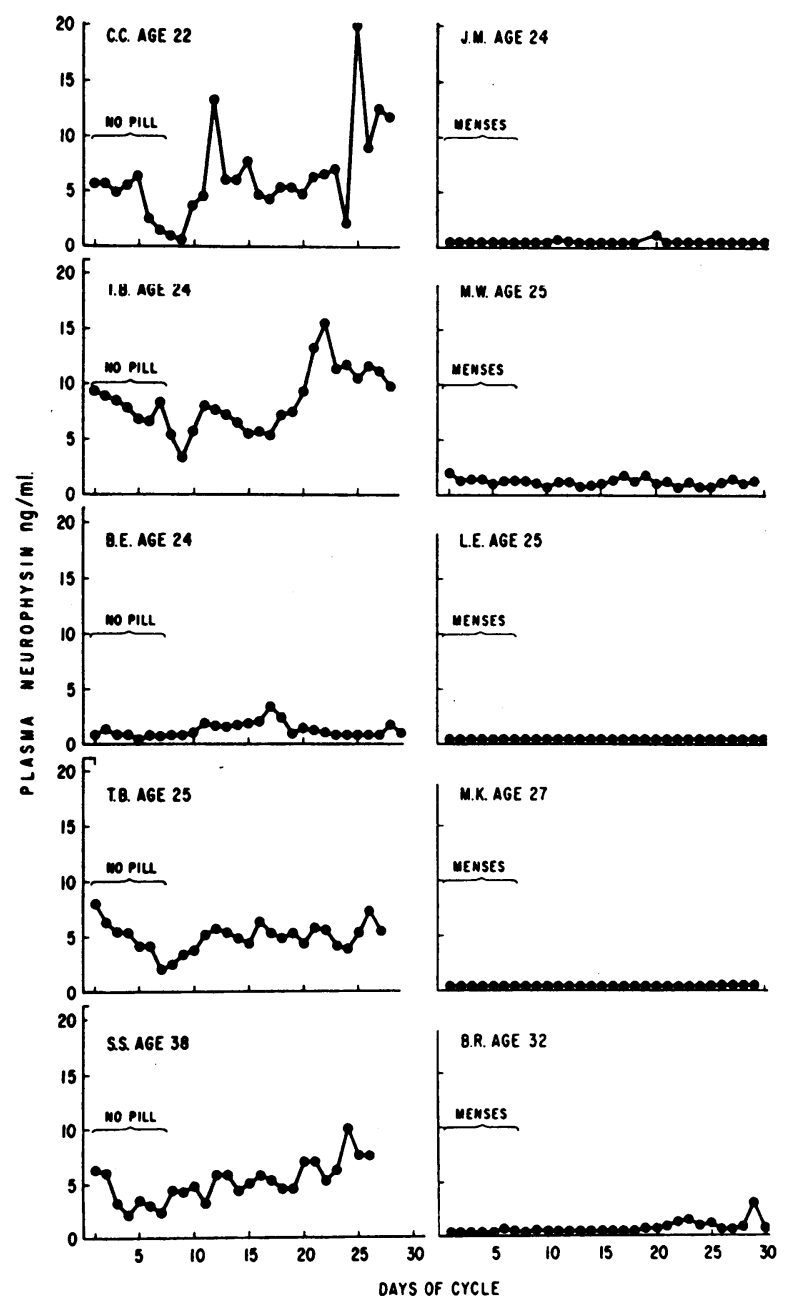

FIgURE 1 Daily plasma neurophysin through a complete cycle in five women on oral contraceptives, left graphs, compared to daily plasma neurophysin in five age-matched controls through a normal menstrual cycle, right graphs.

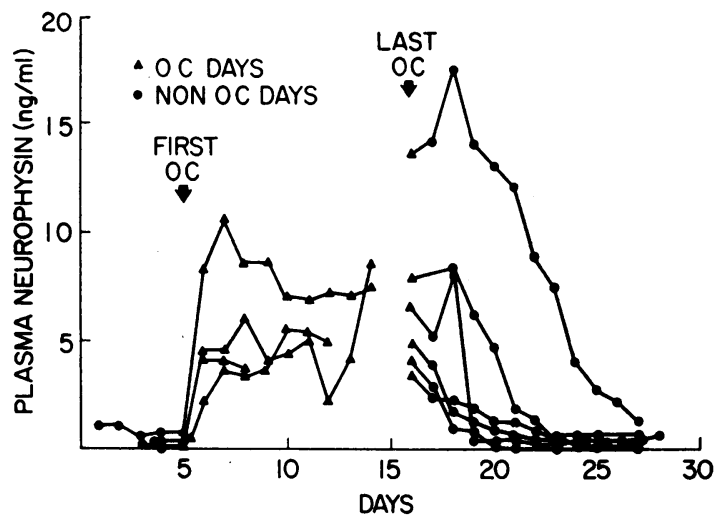

Figure 2 Plasma neurophysin values in four normal women when first starting oral contraceptives (OC) are plotted on the left. The first pill was taken on day 5. Plasma neurophysin values in six women permanently discontinuing oral contraceptives are plotted on the right. The last oral contraceptive was taken as indicated.

neurophysin in women on oral contraceptives was 6.4 $\mathrm{ng} / \mathrm{ml} \pm 4.2 \mathrm{SD}$, nearly 10 -fold greater than the mean value in women with normal cycles. The difference is highly significant $(P<0.001)$. Every woman taking oral contraceptives had at least one sample more than two standard deviations above the normal mean.

Day-to-day variation in plasma neurophysin was studied in five women through a natural menstrual cycle. Little day-to-day variation was found, as illustrated in the graphs on the right of Fig. 1. (Not shown here are the 12 hourly samples in two normal women, which showed little hour-to-hour variation.) This can be contrasted with the higher daily neurophysin values in five approximately age-matched controls on oral contraceptives, left graphs of Fig. 1. Only a single subject, B.E., on oral contraceptives did not show neurophysin values many times greater than the normal controls. Some day-to-day fluctuation in plasma neurophysin is noted in the women on oral contraceptives, which is not seen in the normal controls. However, as many of the values in the control subjects are below the lowest detectable neurophysin concentration $(<0.5 \mathrm{ng} / \mathrm{ml})$, fluctuations at this low level would not be measurable. Four of the women on contraceptives showed some decrease in their plasma neurophysin during the days the oral contraceptives were discontinued, but the 7-day period off contraceptives was not sufficient to establish a plateau in the normal range.

The timing of the change in plasma neurophysin when starting and stopping oral contraceptives was studied in 10 women (Fig. 2). Data from four women starting oral contraceptives are shown on the left. Midmorning samples had low levels of neurophysin until day 5 , when the first contraceptive tablet was taken with breakfast. The mid-morning sample of that day was in the normal range, 
but by the next day, plasma neurophysin was elevated in all women and remained high for the next 3-9 days it was sampled. Values from six other women, who had been on oral contraceptives regularly and who were discontinuing them permanently, are shown on the right. All had elevated plasma neurophysin values which fell to normal 3-11 days after they discontinued the contraceptives.

As most oral contraceptives contain both estrogenic and progestational steroid hormones, it was of interest to determine which of these was responsible for the elevated plasma neurophysin. One of the women who had provided daily plasma samples while discontinuing oral contraceptives and whose plasma neurophysin had been $<0.5 \mathrm{ng} / \mathrm{ml}$ for longer than a week was subsequently treated with diethylstilbestrol (DES) ${ }^{1}$ as a "morningafter" contraception (Fig. 3). DES produced a dramatic rise in plasma neurophysin $16 \mathrm{~h}$ after the first $50-\mathrm{mg}$ dose. This decreased during 4 more days of $50 \mathrm{mg}$ DES/ day. When DES was stopped, plasma neurophysin gradually returned to normal levels over the next 7 days. For comparison, plasma neurophysin in another subject who received 10 days of medroxyprogesterone acetate (MPA) is plotted on the same graph. Neurophysin was undetectable in all samples. This response was confirmed in four male volunteers treated with DES, MPA, and DES + MPA (Fig. 4). $5 \mathrm{mg}$ of DES produced a prompt elevation in plasma neurophysin $12 \mathrm{~h}$ later. The peak value $24 \mathrm{~h}$ after the first dose of DES was sustained, but not further elevated, by a second 5-mg dose. Plasma neurophysin slowly returned to normal over the next 5-7 days. These subjects were also treated with MPA. $72 \mathrm{~h}$ after the start of MPA, no change in neurophysin was measurable in any subject. At this time two doses of DES were given, and $12 \mathrm{~h}$ after the first dose, each subject had responded with an elevation in neurophysin. MPA was continued with the DES, and the peak neurophysin response was not different from the response with DES alone.

\section{DISCUSSION}

The data reported here clearly demonstrate that oral contraceptives cause elevation of plasma neurophysin. This elevation is manifest within the first day of taking oral contraceptives and remains throughout the duration of contraceptive administration. Estrogen rather than progesterone is the stimulus for neurophysin secretion. When estrogen is discontinued, plasma neurophysin values return to normal in 3-11 days. The prompt elevation of plasma neurophysin within several hours of estrogen administration suggests that estrogens may stimulate secretion of preformed neurophysin. This interpretation

${ }^{1}$ Abbreviations used in this paper: DES, diethylstilbestrol; MPA, medroxyprogesterone acetate.

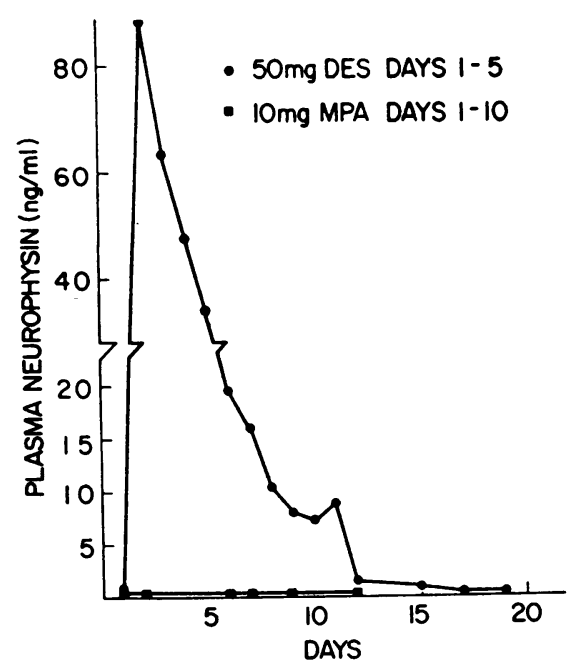

Figlre 3 The effect on plasma neurophysin of the daily administration of $50 \mathrm{mg}$ DES to a normal woman for 5 days (solid circles). This is compared to the effect of the daily administration of $10 \mathrm{mg}$ MPA given to another woman for 10 days (solid squares).

is supported by the response of the subject given a large dose of DES for 5 days. The neurophysin value peaked $24 \mathrm{~h}$ after the first dose and then decreased in spite of continued administration of the same dose of DES (Fig. 3 ). Also, when subjects begin estrogen treatment (Figs. 2 and 4), a plateau of elevated plasma neurophysin is usually reached after the first estrogen dose. This can be contrasted with other effects of estrogens, which are mediated by increased synthesis, e.g., changes in plasma

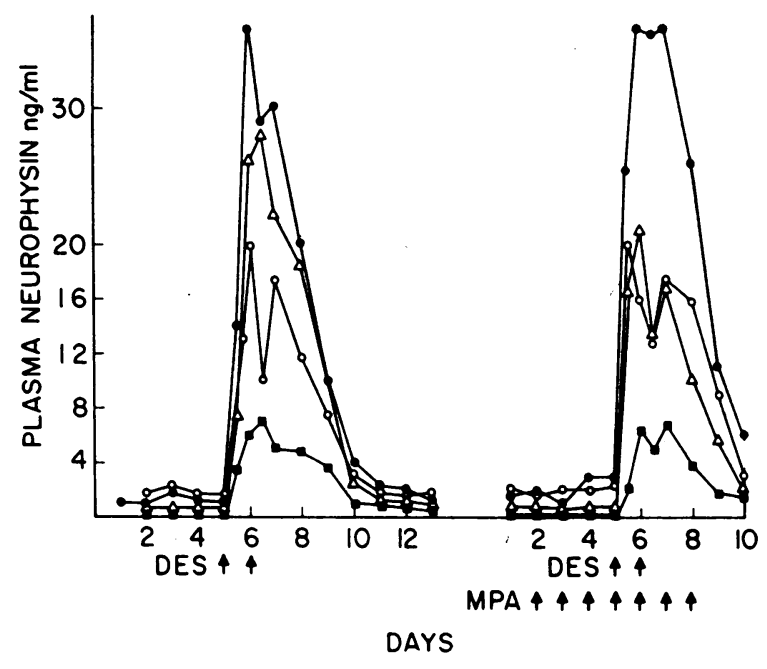

FIGURE 4 Four normal men were given $5 \mathrm{mg}$ of DES, left side of graph, on days 5 and 6 . On the right graph, the same four men were given $10 \mathrm{mg}$ MPA on days 2 through 8. $5 \mathrm{mg}$ DES was given with the MPA on days 5 and 6. Changes in plasma neurophysin are plotted. A different symbol is used for each man. 
binding proteins. For example, thyroid-binding globulin is not maximal until 2 wk of estrogen administration (13).

The clinical significance of elevated plasma neurophysin in women on oral contraceptives will not be certain until the physiologic function of neurophysins is determined. Several studies in animals indicate that plasma neurophysin is increased at times of oxytocin and vasopressin release $(7,10,14)$. Elevated plasma neurophysin in women during estrogen administration may indicate oxytocin and/or vasopressin secretion, but no studies have directly assayed oxytocin or vasopressin in women on oral contraceptives.

The possibility that neurophysins are an integral link in the negative feedback of estrogen on anterior pituitary function might be considered. Anatomic studies have localized neurophysins in the external layer of the median eminence, which is the contact zone for neurosecretion of hypothalamic releasing factors into anterior pituitary blood (9), and recent studies have detected neurophysin in blood from the long portal veins of monkeys (15). In the median eminence, neurophysin is present in tanycytes (9), specialized ependymal cells of the infundibular recess with processes that reach from the third ventricle to the anterior portal blood system (16). These cellular processes have been postulated as an alternate pathway for the secretion of anterior pituitary releasing hormones, i.e., releasing factors might be delivered to the cerebrospinal fluid at the level of the third ventricle, taken up by the tanycytes of the infundibular recess, and transported to hypophyseal portal blood (17). Furthermore, electron microscopy studies of Knowles and Kumar have demonstrated changes in tanycyte granulation, which they attribute to physiologic changes in circulating estrogen (18). These anatomic studies coupled with the present physiologic study, which demonstrates increased neurophysin secretion by estrogen administration, suggests that neurophysins should be investigated for a role in the estrogen feedback on anterior pituitary function.

Regardless of the physiologic consequence of elevated plasma neurophysin in women on oral contraceptives, this observation provides a ready pharmacologic stimulus useful for studying the synthesis and release of neurophysins. As individual neurophysins from human pituitary are isolated and assayed, changes in these neurophysins can be studied by estrogen administration. Thus, this study has demonstrated not only another site of action of oral contraceptives i.e., the neurohypophysis, but has provided a new tool, estrogen administration, for further investigation of the physiologic significance of the carrier proteins, neurophysins.

\section{ACKNOWLEDGMENTS}

These investigations were supported by Clinical Research Center Grant FR-56 from the U. S. Public Health Service and The Population Council, New York City, Grant M72074C.

\section{REFERENCES}

1. Salhanick, H. A., D. M. Kipnis, and R. L. Vandewiele. 1969. Metabolic effects of gonadal hormones and contraceptive steroids. Plenum Publishing Corporation, New York.

2. Elgee, N. J. 1970. Medical aspects of oral contraceptives. Ann. Intern. Med. 72: 409-410.

3. Josimovich, J. B. 1973. Uterine Contraction: Side effects of steroidal contraceptives. John Wiley \& Sons, Inc., New York.

4. Astwood, E. B. 1970. Estrogens and progestins. In The Pharmacologic Basis of Therapeutics. L. S. Goodman and A. Gilman, editors. The Macmillan Company, New York. 4th edition. 1538-1565.

5. Lipsett, M. B. 1971. Problems in contraception. Ann. Intern. Med. 74 : 251-263.

6. Sachs, H., P. Fawcett, Y. Takabatake, and R. Portanova. 1969. Biosynthesis and release of vasopressin and neurophysin. Rec. Prog. Horm. Res. 25: 447-491.

7. Robinson, A. G., E. A. Zimmerman, and A. G. Frantz. 1971. Physiologic investigation of the posterior pituitary binding proteins neurophysin I and II. Metab. (Clin. Exp.). 20: 1148-1155.

8. Cheng, K. W., and H. G. Friesen. 1973. Studies of human neurophysin by radioimmunoassay. J. Clin. Endocrinol. Metab. 36: 553-560.

9. Robinson, A. G., and E. A. Zimmerman. 1973. Cerebrospinal fluid and ependymal neurophysin. J. Clin. Invest. 52 : 1260-1267.

10. Robinson, A. G., and A. G. Frantz. 1973. Radioimmunoassay of posterior pituitary peptides: a review. Metab. (Clin. Exp.). 22 : 1047-1057.

11. Robinson, A. G., D. L. Archer, and L. F. Tolstoi. 1972. Radioimmunoassay of human neurophysin I during parturition. J. Clin. Endocrinol. $37: 645$.

12. Legros, J. J., and P. Franchimont. 1972. Human neurophysine blood levels under normal, experimental and pathological conditions. J. Clin. Endocrinol. 1: 99-113.

13. Dowling, J. T., N. Freinkel, and S. H. Ingbar. 1956. Effect of diethylstilbestrol on the binding of thyroxine in serum. J. Clin. Endocrinol. 16: 1491-1506.

14. Cheng, K. W., and H. G. Friesen. 1970. Physiologic factors regulating secretion of neurophysin. Metab. (Clin. Exp.). 19: 876-890.

15. Zimmerman, E. A., P. W. Carmel, M. K. Husain, M. Ferin, M. Tannenbaum, A. G. Frantz, and A. G. Robinson. 1973. Vasopressin and neurophysin: high concentrations in monkey hypophyseal portal blood. Science (Wash. D. C.). 182 : 925-927.

16. Kumar, T. C. A., and F. Knowles. 1967. A system linking the third ventricle with the pars tuberalis of the rhesus monkey. Nature (Lond.). 215: 54-55.

17. Knigge, K. M., and D. E. Scott. 1970. Structure and function of the median eminence. Am. J. Anat. 129: 223-244.

18. Knowles, F. G. W., and T. C. A. Kumar. 1969. Structural changes, related to reproduction, in the pars tuberalis of the rhusus monkey. Phil. Trans. R. Soc. Lond. Ser. B. Biol. Sci. $256: 357-375$. 